\title{
On non-averaged performance of economic MPC with terminal conditions
}

\author{
Lars Grüne $^{1}$ and Anastasia Panin
}

\begin{abstract}
We present non-averaged and transient performance estimates for economic Model Predictive Control (MPC) schemes with terminal conditions. The results provide a stronger notion of (approximate) optimality than the well known averaged optimality property and improve upon similar results for economic MPC schemes without terminal conditions.
\end{abstract}

\section{INTRODUCTION}

In recent years economic Model Predictive Control (MPC) has seen a large amount of new results. For schemes with terminal conditions (i.e., terminal constraints and possibly terminal costs), bounds on the averaged performance were first given in [2] and further developed in [1], [3]. In case of the existence of an optimal steady state, these results in particular imply optimal averaged performance. Moreover, under a strict dissipativity condition (which is closely related to the existence of an optimal steady state, cf. [10]), asymptotic stability of the optimal steady state for the MPC closed loop could be established, see [6], [1], [3]. In [7], [9], under similar assumptions practical asymptotic stability of the optimal steady state and approximate averaged optimality was shown for economic MPC schemes without terminal conditions.

Infinite horizon averaged optimality, however, is a rather weak concept as trajectories which are optimal on average may behave arbitrarily bad on an arbitrarily long finite time interval before they actually exhibit the desired optimal behavior. For this reason, estimates on the non-averaged infinite horizon performance as well as finite horizon estimates during the transient phase - i.e., estimates about the transient performance - are desirable, too. Transient performance estimates could already be established for economic MPC without terminal conditions in [9]. In this paper we show that under similar assumptions they can also be established for economic MPC with terminal conditions, even with improved estimates for the resulting error terms, cf. Theorem 5.2 and Remark 5.3. Moreover, for the terminal conditioned case we will also be able to give an estimate for the non-averaged infinite horizon performance, $\mathrm{cf}$. Theorem 5.1.

The paper is organized as follows. In Section II we define the problem and in Section III we define the assumptions we impose on the economic MPC scheme. Section IV collects a number of preliminary results which will then

Lars Grüne (lars.gruene@uni-bayreuth.de) and Anastasia Panin are with the Department of Mathematics, University of Bayreuth, 95440 Bayreuth, Germany.

${ }^{1}$ Supported by the German Research Foundation DFG, Grant No. GR1569/13-1. be used to prove the two main theorems in Section V. A numerical example is presented in Section VI and Section VII concludes the paper.

\section{PROBLEM Formulation}

We consider nonlinear discrete time control systems

$$
x(k+1)=f(x(k), u(k))
$$

for $f: X \times U \rightarrow X$, with normed spaces $X$ and $U$ denoting the state and control space, respectively. The solution of system (1) for a control sequence $u=(u(0), u(1), \ldots, u(K-$ 1) $) \in U^{K}$ emanating from the initial value $x$ is denoted by $x_{u}(k, x), k=0, \ldots, K-1$. The set $\mathbb{Y} \subset X \times U$ denotes the admissible state-control pairs and $\mathbb{X}:=\{x \in$ $X \mid$ there exists $u \in U$ with $(x, u) \in \mathbb{Y}\}$ is the induced set of admissible states. For a given initial value $x \in \mathbb{X}$, a control sequence $u \in \mathbb{U}^{K}$ is called admissible if $\left(x_{u}(k, x), u(k)\right) \in$ $\mathbb{X}$ holds for all time instants $k=0, \ldots, K-1$. The set of all admissible control sequences is denoted by $\mathbb{U}^{K}(x)$. For the infinite horizon case $u=(u(0), u(1), \ldots) \in U^{\infty}$ we define the sets $\mathbb{U}^{\infty}$ and $\mathbb{U}^{\infty}(x)$ analogously. For a set $B \subset X$ we define the set of controls

$$
\mathbb{U}_{B}^{K}(x):=\left\{u \in \mathbb{U}^{K}(x) \mid x_{u}(N, x) \in B\right\}
$$

which steer the initial condition into $B$ after $N$ steps. We will use this concept both for terminal constraint sets $B=\mathbb{X}_{0}$ and for balls $B=\bar{B}_{\kappa}(\tilde{x}):=\{x \in X \mid\|x-\tilde{x}\| \leq \kappa\}$.

For a given stage cost $\ell: \mathbb{Y} \rightarrow \mathbb{R}$, a terminal cost $V_{f}:$ $\mathbb{X}_{0} \rightarrow \mathbb{R}$ defined on a terminal constraint set $\mathbb{X}_{0}$, a horizon $N \in \mathbb{N}$ and all $x \in \mathbb{X}$ and $u \in \mathbb{U}_{\mathbb{X}_{0}}^{N}(x)$ we define the finite horizon cost functional

$$
J_{N}(x, u):=\sum_{k=0}^{N-1} \ell\left(x_{u}(k, x), u(k)\right)+V_{f}\left(x_{u}(N, x)\right),
$$

and the corresponding optimal value function

$$
V_{N}(x):=\inf _{u \in \mathbb{U}_{\mathbb{X}_{0}}^{N}(x)} J_{N}(x, u) .
$$

We note that $V_{N}$ is defined on the feasible set $\mathbb{X}_{N}:=\{x \in$ $\left.X \mid \mathbb{U}_{\mathbb{X}_{0}}^{N}(x) \neq \emptyset\right\}$.

For $x \in \mathbb{X}$ and $u \in \mathbb{U}^{N}(x)$ we also define the unconditioned functional (i.e., without terminal constraints and cost)

$$
J_{N}^{u c}(x, u):=\sum_{k=0}^{N-1} \ell\left(x_{u}(k, x), u(k)\right)
$$

and the corresponding optimal value function

$$
V_{N}^{u c}(x):=\inf _{u \in \mathbb{U}^{N}(x)} J_{N}^{u c}(x, u) .
$$


Moreover, we define the (unconditioned) infinite horizon functional $J_{\infty}^{u c}(x, u):=\limsup _{N \rightarrow \infty} J_{N}^{u c}(x, u)$ and the corresponding optimal value function $V_{\infty}^{u c}(x):=$ $\inf _{u \in \mathbb{U}^{\infty}(x)} J_{\infty}^{u c}(x, u)$ which is defined for all $x \in \mathbb{X}_{\infty}:=$ $\left\{x \in \mathbb{X} \mid \mathbb{U}^{\infty}(x) \neq \emptyset\right\}$.

In the sequel we assume that for all $N \in \mathbb{N}$ and $x \in \mathbb{X}_{N}$ there is an optimal control sequence $u_{N, x}^{\star} \in$ $\mathbb{U}_{\mathbb{X}_{0}}^{N}(x)$, i.e., a control sequence for which the equality $V_{N}(x)=J_{N}\left(x, u_{N, x}^{\star}\right)$ holds. We remark that optimal control sequences need not be unique; in this case $u_{N, x}^{\star}$ denotes one of the possible optimal control sequences.

Using the optimal control problem (2), (3), we now define the model predictive control (MPC) scheme we analyze in this paper. Fixing an optimization horizon $N \in \mathbb{N}$, at each time instant $n$ we perform the following steps:

1) Measure the current state $x=x(n)$ of the system.

2) Solve the optimization problem of minimizing $J_{N}(x, u)$ with respect to $u \in \mathbb{U}_{\mathbb{X}_{0}}^{N}(x)$ and denote the resulting optimal control sequence by $u_{N, x}^{\star}$.

3) Apply the first element of $u_{N, x}^{\star}$ as a feedback control value until the next time instant, i.e., define the feedback law $\mu_{N}(x):=u_{N, x}^{\star}(0)$.

The resulting MPC closed loop system is given by $x(n+1)=$ $f\left(x(n), \mu_{N}(x(n))\right)$. Trajectories of this system with initial value $x \in \mathbb{X}$ will be denoted by $x_{\mu_{N}}(n, x)$

As the MPC feedback law is derived from minimizing (2), questions about the optimality properties of the closed loop naturally arise. In this paper we will investigate

$$
J_{K}^{c l}\left(x, \mu_{N}\right):=\sum_{n=0}^{K-1} \ell\left(x_{\mu_{N}}(n, x), \mu_{N}\left(x_{\mu_{N}}(n, x)\right)\right)
$$

for arbitrary $K \in \mathbb{N}$ as well as the non-averaged infinite horizon performance measure $J_{\infty}^{c l}\left(x, \mu_{N}\right)=$ $\limsup _{K \rightarrow \infty} J_{K}^{c l}\left(x, \mu_{N}\right)$. We emphasize that this performance criterion yields a tighter notion of optimality than its averaged counterpart $\lim _{\sup _{K \rightarrow \infty}} \frac{1}{K} J_{K}^{c l}\left(x, \mu_{N}\right)$ which is often used in the economic MPC literature.

Throughout the paper we will make use of the following classes of comparison functions

$$
\begin{aligned}
& \mathcal{L}:=\left\{\begin{array}{l|l}
\delta: \mathbb{R}_{0}^{+} \rightarrow \mathbb{R}_{0}^{+} & \begin{array}{l}
\delta \text { continuous and decreasing } \\
\text { with } \lim _{k \rightarrow \infty} \delta(k)=0
\end{array}
\end{array}\right\}, \\
& \mathcal{K}:=\left\{\begin{array}{l|l}
\alpha: \mathbb{R}_{0}^{+} \rightarrow \mathbb{R}_{0}^{+} & \begin{array}{l}
\alpha \text { continuous and strictly } \\
\text { increasing with } \alpha(0)=0
\end{array}
\end{array}\right\}, \\
& \mathcal{K}_{\infty}:=\{\alpha \in \mathcal{K} \mid \alpha \text { unbounded }\}, \\
& \mathcal{K} \mathcal{L}:=\left\{\begin{array}{l|l}
\beta: \mathbb{R}_{0}^{+} \times \mathbb{R}_{0}^{+} \rightarrow \mathbb{R}_{0}^{+} & \begin{array}{l}
\beta \text { continuous }, \\
\beta(\cdot, t) \in \mathcal{K}, \beta(r, \cdot) \in \mathcal{L}
\end{array}
\end{array}\right\} .
\end{aligned}
$$

Moreover, we will use the dynamic programming principle for $V_{N}$ from (3) which for all $k=1, \ldots, N-1$ reads

$$
V_{N}(x)=\inf _{u \in \mathbb{U}_{\mathbb{X}_{N-k}^{k}}^{k}(x)}\left\{J_{k}^{u c}(x, u)+V_{N-k}\left(x_{u}(k, x)\right)\right\},
$$

cf. [8, Theorem 3.15].

\section{Assumptions}

In this section we define the precise assumptions on the ingredients of the MPC scheme under consideration and state some immediate consequences. Our assumptions are identical to those found in the economic MPC literature in order to ensure existence and asymptotic stability of an optimal steady state [1], [3].

Assumption 3.1 (compactness and continuity): The constraint set $\mathbb{Y} \subset \mathbb{X} \times \mathbb{U}$ is compact and the maps $\ell: \mathbb{X} \times \mathbb{U} \rightarrow \mathbb{R}$ and $f: \mathbb{X} \times \mathbb{U} \rightarrow \mathbb{X}$ are continuous.

Under this assumption, the constant $M:=\sup _{x, y \in \mathbb{X}}\|x-y\|$ is finite and the following lemma holds.

Lemma 3.2: If Assumption 3.1 holds, then there exists an optimal equilibrium, i.e., a pair $x^{e} \in \mathbb{X}, u^{e} \in \mathbb{U}$ with $f\left(x^{e}, u^{e}\right)=x^{e}$ such that

$$
\ell\left(x^{e}, u^{e}\right)=\inf \{\ell(x, u) \mid(x, u) \in \mathbb{Y}, f(x, u)=x\} .
$$

Proof: Since pre-images of closed sets under continuous mappings are closed, the set $\{(x, u) \in \mathbb{Y} \mid f(x, u)=x\}$ is closed, hence compact and thus the continuous function $\ell$ attains a minimum.

Assumption 3.3 (terminal conditions): (a) The terminal cost $V_{f}$ satisfies

$$
\left|V_{f}(x)-V_{f}\left(x^{e}\right)\right| \leq \gamma_{f}\left(\left\|x-x^{e}\right\|\right)
$$

for some $\gamma_{f} \in \mathcal{K}_{\infty}$ and all $x \in \mathbb{X}_{f}$ and for each $x \in \mathbb{X}_{f}$ there exists $u \in \mathbb{U}$ with $f(x, u) \in \mathbb{X}_{f}$ and

$$
V_{f}(f(x, u)) \leq V_{f}(x)-\ell(x, u)+\ell\left(x^{e}, u^{e}\right)
$$

(b) There exists $N_{0} \in \mathbb{N}$ and $\eta>0$ such that $\mathbb{X}_{N_{0}}$ contains the ball $B_{\eta}\left(x^{e}\right)$.

We note that Assumption 3.3(a) is satisfied in case of equilibrium terminal constraints, i.e., when setting $\mathbb{X}_{f}=\left\{x^{e}\right\}$ and $V_{f} \equiv 0$. Standard MPC arguments show that under Assumption 3.3(a) the feasible sets satisfy the inclusion $\mathbb{X}_{N_{0}} \subseteq \mathbb{X}_{N_{1}}$ whenever $N_{0} \leq N_{1}$. Assumption 3.3(b) is imposed in order to avoid technicalities which arise when the domain of definition of the MPC controller does not contain a neighbourhood of $x^{e}$.

Assumption 3.4 (strict dissipativity): There exists a storage function $\lambda: X \rightarrow \mathbb{R}$ and a function $\alpha \in \mathcal{K}_{\infty}$ such that for all $(x, u) \in \mathbb{Y}$ the inequality

$$
\ell(x, u)-\ell\left(x^{e}, u^{e}\right)+\lambda(x)-\lambda(f(x, u)) \geq \alpha\left(\left\|x-x^{e}\right\|\right)
$$

holds. Moreover, $\lambda\left(x^{e}\right)=0$ and there exists $\gamma_{\lambda} \in \mathcal{K}_{\infty}$ with

$$
|\lambda(x)| \leq \gamma_{\lambda}\left(\left\|x-x^{e}\right\|\right)
$$

We note that the assumption $\lambda\left(x^{e}\right)=0$ can be made without loss of generality.

Definition 3.5: The function

$$
\tilde{\ell}(x, u):=\ell(x, u)-\ell\left(x^{e}, u^{e}\right)+\lambda(x)-\lambda(f(x, u))
$$

is called the modified (or rotated) stage cost and the function

$$
\widetilde{V}_{f}(x):=V_{f}(x)+\lambda(x)
$$


is called the modified (or rotated) terminal cost. Analogously to (2)-(5) we define $\widetilde{J}_{N}, \widetilde{V}_{N}, \widetilde{J}_{N}^{u c}$ and $\widetilde{V}_{N}^{u c}$, respectively, using $\tilde{\ell}$ and $\widetilde{V}_{f}$ instead of $\ell$ and $V_{f}$.

It is an easy exercise to check that the equality $\tilde{\ell}\left(x^{e}, u^{e}\right)=$ 0 and the inequality $\widetilde{V}_{f}(f(x, u)) \leq \widetilde{V}_{f}(x)-\tilde{\ell}(x, u)$ hold for all $(x, u) \in \mathbb{Y}$. Moreover, for any $x \in \mathbb{X}_{N}$ and $u \in \mathbb{U}^{N}(x)$ one easily checks the identity $\widetilde{J}_{N}(x, u)=J_{N}(x, u)+\lambda(x)-$ $N \ell\left(x^{e}, u^{e}\right)$ which implies that the optimal trajectories for $J_{N}$ and $\widetilde{J}_{N}$ coincide and that the optimal value functions satisfy

$$
\widetilde{V}_{N}(x)=V_{N}(x)+\lambda(x)-N \ell\left(x^{e}, u^{e}\right) .
$$

Moreover, non-negativity of $\tilde{\ell}$ implies $\widetilde{V}_{N}(x) \geq 0$ and from $\widetilde{J}_{N}\left(x^{e}, u\right)=0$ for $u \equiv u^{e}$ we thus obtain $\widetilde{V}_{N}\left(x^{\bar{e}}\right)=0$. Using (6) and $\lambda\left(x^{e}\right)=0$ we can conclude $V_{N}\left(x^{e}\right)=N \ell\left(x^{e}, u^{e}\right)$.

For the unconstrained functional we obtain

$\widetilde{J}_{N}^{u c}(x, u)=J_{N}^{u c}(x, u)+\lambda(x)-\lambda\left(x_{u}(N, x)\right)-N \ell\left(x^{e}, u^{e}\right)$,

implying that because $\lambda\left(x_{u}(N, x)\right)$ depends on $u$ the optimal trajectories for $J_{N}^{u c}$ and $\widetilde{J}_{N}^{u c}$ do not coincide, in general.

Assumption 3.6: (bound on $V_{N}$ ) There exists $\gamma_{V} \in \mathcal{K}_{\infty}$ such that for each $N \in \mathbb{N}$ and each $x \in \mathbb{X}_{N}$ it holds that

$$
\left|V_{N}(x)-V_{N}\left(x^{e}\right)\right| \leq \gamma_{V}\left(\left\|x-x^{e}\right\|\right) .
$$

We remark that for $\mathbb{X}_{f}=\left\{x^{e}\right\}$ Assumption 3.6 follows from the controllability condition [3, Assumption 2, 2)] and continuity of $f$ and $\ell$ while if $\mathbb{X}_{f}$ is a neighborhood of $x^{e}$ then it follows from the proof of Theorem 15 in [1] and the relation between $V_{N}$ and $\widetilde{V}_{N}$.

\section{PREliminary Results}

In this section we collect a number of preliminary results which will be used in the proofs of our main results in the next section. The first result states that under the assumptions introduced in the previous section the equilibrium $x^{e}$ is asymptotically stable. for the MPC closed loop.

Theorem 4.1: Under Assumptions 3.1, 3.3, 3.4 and 3.6 the equilibrium $x^{e}$ is asymptotically stable for the MPC closed loop with domain of attraction $\mathbb{X}_{N}$ and Lyapunov function $\widetilde{V}_{N}$ satisfying

$$
\widetilde{V}_{N}\left(f\left(x, \mu_{N}(x)\right)\right) \leq \widetilde{V}_{N}(x)-\tilde{\ell}\left(x, \mu_{N}(x)\right) .
$$

Particularly, there is $\beta \in \mathcal{K} \mathcal{L}$ such that for all $x \in \mathbb{X}_{N}$ and all $k \in \mathbb{N}$ the inequality $\left\|x_{\mu_{N}}(k, x)-x^{e}\right\| \leq \beta\left(\left\|x-x^{e}\right\|, k\right)$ holds.

Proof: For equilibrium terminal constraints this follows from [3, Theorem 2] and for the general case the assertion is proved in [1, Theorem 15].

We note that by (6), (8) implies the inequality

$$
V_{N}\left(f\left(x, \mu_{N}(x)\right)\right) \leq V_{N}(x)-\ell\left(x, \mu_{N}(x)\right)+\ell\left(x^{e}, u^{e}\right)
$$

for the non-rotated problem.

The next lemma provides upper and lower bounds on the infinite horizon optimal value function $V_{\infty}^{u c}$.
Lemma 4.2: Assume $\ell\left(x^{e}, u^{e}\right)=0$ and let Assumptions 3.1, 3.3, 3.4 and 3.6 hold. Then there is $C>0$ such that the inequalities

$$
-C \leq V_{\infty}^{u c}(x) \leq \gamma_{V}\left(\left\|x-x^{e}\right\|\right)
$$

hold for all $x \in \mathbb{X}_{\infty}$ with $\gamma_{V}$ from Assumption 3.6.

Proof: Using the control sequence $u(k)=\mu_{N}\left(x_{\mu_{N}}(k, x)\right)$ induced by the closed loop, from (9) with $\ell\left(x^{e}, u^{e}\right)=0$ for any $K>0$ we obtain

$$
\begin{aligned}
J_{K}^{u c}(x, u) & =\sum_{k=0}^{K-1} \ell\left(x_{u}(k, x), u_{k}(x)\right) \\
& \leq V_{N}(x)-V_{N}\left(x_{u}(K, x)\right) .
\end{aligned}
$$

By asymptotic stability of $x^{e}$ for this solution we obtain $x_{u}(K, x) \rightarrow x^{e}$ and thus, since $V_{N}\left(x^{e}\right)=N \ell\left(x^{e}, u^{e}\right)=0$, Assumption 3.6 yields $V_{N}\left(x_{u}(K, x)\right) \rightarrow 0$ as $K \rightarrow \infty$. Using Assumption 3.6 and $V\left(x^{e}\right)=0$ once more, this implies

$$
V_{\infty}^{u c}(x) \leq \limsup _{K \rightarrow \infty} J_{K}^{u c}(x, u) \leq V_{N}(x) \leq \gamma_{V}\left(\left\|x-x^{e}\right\|\right) .
$$

On the other hand, the fact that $\widetilde{J}_{N}^{u c}(x, u) \geq 0$ and again (6) and the boundedness of $\lambda$ imply $J_{N}^{u c}(x, u) \geq-C$ for some $C \geq 0$ and all $x, u$ and $N$. This implies $V_{\infty}^{u c}(x) \geq$ $-C$.

The next theorem establishes a property of optimal trajectories called the turnpike property. The version of the turnpike property presented here is the discrete time version of the continuous time formulation found in [4].

Theorem 4.3: Let Assumptions 3.1, 3.3, 3.4 and 3.6 hold. Then there exist a $C>0$ such that for each $x \in \mathbb{X}, \delta>0$ and $K \in \mathbb{N}$, each control sequence $u \in U^{K}(x)$ satisfying $J_{K}^{u c}(x, u) \leq K \ell\left(x^{e}, u^{e}\right)+\delta$ and each $\varepsilon>0$ the value $Q_{\varepsilon}:=$ $\#\left\{k \in\{0, \ldots, K-1\} \mid\left\|x_{u}(k, x)-x^{e}\right\| \leq \varepsilon\right\}$ satisfies the inequality $Q_{\varepsilon} \geq K-(\delta+C) / \rho(\varepsilon)$.

Proof: See [7, Theorem 5.3].

We remark that under stronger assumptions an exponential relation between $\varepsilon$ an $K$ of the form $\varepsilon=\theta^{K}$ for some $\theta \in(0,1)$ can be obtained, cf. [5]. An extension of Theorem 4.3 to infinite horizon trajectories is provided in the following corollary.

Corollary 4.4: Assume $\ell\left(x^{e}, u^{e}\right)=0$ and let Assumptions 3.1, 3.3, 3.4 and 3.6 hold. Then there exists $\sigma \in \mathcal{L}$ such that for any $x \in \mathbb{X}_{\infty}$, any $u \in \mathbb{U}^{\infty}(x)$ with $J_{\infty}^{u c}(x, u) \leq$ $V_{\infty}^{u c}(x)+1$ and any $K \in \mathbb{N}$ and $p \in \mathbb{N}$ there is $k \in \mathbb{N}$ with $p \leq k \leq K+p$ such that $\left\|x_{u}(k, x)-x^{e}\right\| \leq \sigma(K)$.

Proof: We first show the property for $p=0$. Since by Assumption 3.1 and Lemma 4.2 the function $V_{\infty}^{u c}$ is bounded by $\gamma_{V}(M)$ for $M:=\max _{x, y \in \mathbb{X}}\|x-y\|$, the assumption implies $J_{\infty}^{u c}(x, u) \leq \gamma_{V}(M)+1$. Moreover, again Lemma 4.2 implies $J_{\infty}^{u c}(x, u) \geq V_{\infty}^{u c}(x) \geq-C$ for all $x$ and $u$. This implies

$$
\begin{aligned}
\gamma_{V}(M)+1 & \geq J_{\infty}^{u c}(x, u) \\
& =J_{K}^{u c}(x, u)+J_{\infty}^{u c}\left(x_{u}(N, x), u(N+\cdot)\right) \\
& \geq J_{K}^{u c}(x, u)+-C
\end{aligned}
$$


and thus for any $K$ the value of the functional $J_{K}^{u c}(x, u)$ satisfies the assumption of Theorem 4.3 with $\delta=C+\gamma_{V}(M)+$ 1 ; without loss of generality we can assume that this $C$ and the constant $C$ from Theorem 4.3 are identical. Applying this theorem with $\varepsilon=\delta(K)=\rho^{-1}\left(\left(2 C+\gamma_{V}(M)+1\right) /(K-1)\right)$ one checks that $Q_{\varepsilon} \geq 1$ which shows the assertion for $p=0$.

For arbitrary $p \in \mathbb{N}$ we can use that $J_{\infty}^{u c}(x, u) \leq V_{\infty}^{u c}(x)+$ 1 implies $J_{\infty}^{u c}\left(x_{u}(p, x), u(p+\cdot)\right) \leq V_{\infty}^{u c}\left(x_{u}(p, x)\right)+1$. Replacing $x$ by $x_{u}(p, x)$ in the proof, above, shows the desired claim.

For sequences $p_{j} \rightarrow \infty$ and $K_{j} \rightarrow \infty$ (implying $\sigma\left(K_{j}\right) \rightarrow$ $0)$, the corollary implies there exists a sequence $k_{j} \rightarrow \infty$ with $x_{u}\left(k_{j}, x\right) \rightarrow x^{e}$ as $j \rightarrow \infty$. Using this fact we can improve the lower bound on $V_{\infty}^{u c}$ from Lemma 4.2.

Lemma 4.5: Assume $\ell\left(x^{e}, u^{e}\right)=0$ and let Assumptions $3.1,3.3,3.4$ and 3.6 hold. Then the inequality $V_{\infty}^{u c}(x) \geq$ $-\lambda(x)$ holds for all $x \in \mathbb{X}_{\infty}$.

Proof: Let $u \in \mathbb{U}^{\infty}(x)$ be such that $J_{\infty}^{u c}(x, u) \leq V_{\infty}^{u c}(x)+\varepsilon$ for an $\varepsilon \in(0,1)$. As explained above, Corollary 4.4 implies that there exists a sequence $K_{j} \rightarrow \infty$ with $x_{u}\left(K_{j}, x\right) \rightarrow x^{e}$ as $j \rightarrow \infty$. The definition of $V_{\infty}^{u c}$ and (7) then imply that

$$
\begin{aligned}
& V_{\infty}^{u c}(x)+\varepsilon \geq \limsup _{j \rightarrow \infty} J_{k_{j}}^{u c}(x, u) \\
& =\limsup _{j \rightarrow \infty}(-\lambda(x)+\underbrace{\widetilde{J}_{k_{j}}^{u c}(x, u)}_{\geq 0}+\underbrace{\lambda\left(x_{u}\left(k_{j}, x\right)\right.}_{\rightarrow \lambda\left(x^{e}\right)=0}) \geq-\lambda(x) .
\end{aligned}
$$

This implies the assertion since $\varepsilon>0$ was arbitrary.

Our final preparatory result concerns the optimal value of the problem with control functions $u$ which steer a given initial value to the closed ball $\bar{B}_{\kappa}\left(x^{e}\right)$ with radius $\kappa>0$ around $x^{e}$, i.e., $u \in \mathbb{U}_{\bar{B}_{\kappa}\left(x^{e}\right)}^{K}(x)$. We remark that for $x \in \mathbb{X}_{N}$ Theorem 4.1 implies that for $K$ with $\beta\left(\left\|x-x^{e}\right\|, K\right) \leq$ $\kappa$ the control $u$ obtained from the MPC feedback law via $u(k)=\mu_{N}\left(x_{\mu_{N}}(k, x)\right)$ is contained in $\mathbb{U}_{B_{\kappa}\left(x^{e}\right)}^{K}(x)$. This, in particular, shows that this set is nonempty for sufficiently large $K$.

The next lemma shows that the infimum of $J_{K}^{u c}(x, u)$ over $u \in \mathbb{U} \bar{B}_{\kappa}\left(x^{e}\right)(x)$ and the corresponding approximately optimal trajectories behave similar to those for the infinite horizon problem.

Lemma 4.6: Let Assumptions 3.1, 3.3, 3.4 and 3.6 hold and fix $\kappa_{0}>0$. Then for any $\kappa \in\left(0, \kappa_{0}\right]$, any $x \in \mathbb{X}$ and $K_{0} \in \mathbb{N}$ minimal with $\beta\left(\left\|x-x^{e}\right\|, K_{0}\right) \leq \kappa$ for $\beta$ from Theorem 4.1, the following holds.

(a) For all $K \geq K_{0}$ the inequality

$\inf _{u \in \mathbb{U}_{\bar{B}_{\kappa}\left(x^{e}\right)}(x)} J_{K}^{u c}(x, u)-K \ell\left(x^{e}, u^{e}\right) \leq \gamma_{V}\left(\left\|x-x^{e}\right\|\right)+\gamma_{V}(\kappa)$

holds with $\gamma_{V} \in \mathcal{K}_{\infty}$ from Assumption 3.6.

(b) For all $K \in \mathbb{N}$ with $\mathbb{U}_{B_{\kappa}\left(x^{e}\right)}^{K}(x) \neq \emptyset$ the inequality

$$
\lambda(x)-\gamma_{\lambda}(\kappa) \leq \inf _{u \in \mathbb{U}_{\bar{B}_{\kappa}\left(x^{e}\right)}(x)} J_{K}^{u c}(x, u)-K \ell\left(x^{e}, u^{e}\right)
$$

hold with $\gamma_{\lambda}$ from Assumption 3.4.

(c) There exists $\sigma \in \mathcal{L}$ such that for all $K \geq K_{0}$, all $P \in \mathbb{N}$, any $u \in \mathbb{U}_{\bar{B}_{\kappa}\left(x^{e}\right)}^{K}(x)$ with $J_{K}^{u c}(x, u) \leq$ $\inf _{u \in \mathbb{U}_{\bar{B}_{K}(x)} e^{e}}(x) J_{K}^{u c}(x, u)+1$ there is $k \leq \min \{P, K-1\}$ such that $\left\|x_{u}(k, x)-x^{e}\right\| \leq \delta(\min \{P, K-1\})$.

Proof: (a) The proof of this inequality works similar to the first part of the proof of Lemma 4.2. We choose the control $u$ obtained from the MPC feedback law via $u(k)=$ $\mu_{N}\left(x_{\mu_{N}}(k, x)\right)$. As in the proof of Lemma 4.2, from (9) now with $\ell\left(x^{e}, u^{e}\right) \neq 0$ - for this $u$ we get

$$
J_{K}^{u c}(x, u) \leq V_{N}(x)-V_{N}\left(x_{u}(K, x)\right)+K \ell\left(x^{e}, u^{e}\right)
$$

and from Assumption 3.6 and $\left\|x_{u}(K, x)-x^{e}\right\|<\kappa$ we obtain the assertion.

(b) For this inequality we proceed similarly as in the proof of Lemma 4.5, again now taking into account $\ell\left(x^{e}, u^{e}\right) \neq$ 0 . Let $\varepsilon>0$ and take a control $u \in \mathbb{U}_{\bar{B}_{\kappa}\left(x^{e}\right)}^{K}(x)$ with $\inf _{u \in \mathbb{U}_{\bar{B}_{\kappa}\left(x^{e}\right)}(x)} J_{K}^{u c}(x, u) \geq J_{K}^{u c}(x, u)+\varepsilon$. Then

$$
\begin{aligned}
& \inf _{u \in \mathbb{U} \frac{K}{\bar{B}_{\kappa}\left(x^{e}\right)}(x)} J_{K}^{u c}(x, u)+\varepsilon \geq J_{K}^{u c}(x, u) \\
& =-\lambda(x)+\underbrace{\widetilde{J}_{K}^{u c}(x, u)}_{\geq 0}+\underbrace{\lambda\left(x_{u}(K, x)\right)}_{\geq-\gamma_{\lambda}(\kappa)}+K \ell\left(x^{e}, u^{e}\right) \\
& \geq \lambda\left(x^{e}\right)-\gamma_{\lambda}(\kappa)+K \ell\left(x^{e}, u^{e}\right) .
\end{aligned}
$$

This implies (b) since $\varepsilon>0$ was arbitrary.

(c) The assumptions and (a) imply that Theorem 4.3 can be applied with $\delta=\gamma\left(\left\|x-x^{e}\right\|\right)+\gamma(\kappa)+1$ which can be bounded by a constant $C$ for all $x \in \mathbb{X}$ and all $\kappa \in\left(0, \kappa_{0}\right]$. Without loss of generality we may assume that this $C$ coincides with the constant $C$ from Theorem 4.3. Hence, applying this theorem with $\varepsilon=\sigma(\min \{P, K-1\})$ with $\sigma(k)=$ $\alpha^{-1}(2 C / k)$, one checks that $Q_{\varepsilon} \geq \max \{K-P, 1\}$, implying that there exists at least one $k \in\{0, \ldots, \min \{P, K-1\}\}$ with $\left\|x_{u}(k, x)-x^{e}\right\| \leq \varepsilon$.

\section{Main Results}

We now have all the tools to prove our two main theorems. The first theorem gives an upper bound for the nonaveraged infinite horizon performance of the MPC closed loop trajectory. Taking into account the inequality $V_{\infty}^{u c}(x) \leq$ $J_{\infty}^{c l}\left(x, \mu_{N}\right)$ which follows immediately from the definition of these functions, the theorem shows that economic MPC delivers an approximately (non-averaged) infinite horizon optimal closed loop solution for which the approximation error tends to 0 as the horizon $N$ tends to infinity.

Theorem 5.1: Assume $\ell\left(x^{e}, u^{e}\right)=0$ and let Assumptions 3.1, 3.3, 3.4 and 3.6 hold. Then there exists $\delta \in \mathcal{L}$ such that the inequalities

$$
J_{\infty}^{c l}\left(x, \mu_{N}\right) \leq V_{N}(x) \leq V_{\infty}^{u c}(x)+\delta(N)
$$

hold for all $x \in \mathbb{X}_{N}$.

Proof: In order to prove the first inequality, from (9) we obtain $\ell\left(x, \mu_{N}(x)\right) \leq V_{N}(x)-V_{N}\left(f\left(x, \mu_{N}(x)\right)\right)$. This implies for any $K \in \mathbb{N}$

$$
\begin{aligned}
J_{K}^{c l}\left(x, \mu_{N}\right) & =\sum_{k=0}^{K-1} \ell\left(x_{\mu_{N}}(k, x), \mu_{N}\left(x_{\mu_{N}}(k, x)\right)\right) \\
& =V_{N}(x)-V_{N}\left(x_{\mu_{N}}(K, x)\right)
\end{aligned}
$$


Now from the asymptotic stability we know that $\left.\| x_{\mu_{N}}(k, x)-x^{e}\right) \| \leq \beta\left(\left\|x-x^{e}\right\|, k\right) \leq \beta(M, k)=: \sigma(k)$, where $M:=\max _{x, y \in \mathbb{X}}\|x-y\|$. Note that $\sigma \in \mathcal{L}$. Moreover, as observed after (6) we have $V_{N}\left(x^{e}\right)=N \ell\left(x^{e}, u^{e}\right)=0$ and from Assumption 3.6 we know the existence of $\gamma_{V} \in \mathcal{K}$ with $\left|V_{N}(x)\right|=\left|V_{N}(x)-V_{N}\left(x^{e}\right)\right| \leq \gamma_{V}\left(\left\|x-x^{e}\right\|\right)$ for all $x \in X$. Together this yields

$$
\left|V_{N}\left(x_{\mu_{N}}(K, x)\right)\right| \leq \gamma_{V}(\sigma(K))
$$

Since $\gamma_{V}(\sigma(K)) \rightarrow 0$ for $K \rightarrow \infty$, this inequality together with (10) yields the first inequality by letting $K \rightarrow \infty$.

For the second inequality, we use Corollary 4.4. We note that it is sufficient to prove the inequality for all sufficiently large $N$, because by boundedness of $V_{N}$ and $V_{\infty}^{u c}$, for small $N$ the inequality can always be satisfied by choosing $\delta(N)$ sufficiently large without violating the requirement $\delta \in \mathcal{L}$. We now pick $N_{0}$ and $\eta$ from Assumption 3.3(b), fix $0<\varepsilon<$ 1 and pick an admissible control $u_{\varepsilon}$ satisfying $J_{\infty}^{u c}\left(x, u_{\varepsilon}\right) \leq$ $V_{\infty}^{u c}(x)+\varepsilon$. Then for $N \geq 2 N_{0}$ we apply Corollary 4.4 with $K=\lfloor N / 2\rfloor$. We thus obtain the existence of $k \in$ $\{0, \ldots, K-1\}$ such that $\left\|x_{u_{\varepsilon}}(k, x)-x^{e}\right\| \leq \sigma(K) \leq \sigma\left(N_{0}\right)$, implying $x_{u}(k, x) \in \mathbb{X}_{N_{0}} \subseteq \mathbb{X}_{N_{1}}$ and thus $u_{\varepsilon} \in \mathbb{U}_{\mathbb{X}_{N_{1}}}^{k}(x)$ for all $N_{1} \geq N_{0}$. Particularly, this holds for $N_{1}=N-$ $k$, implying $u_{\varepsilon} \in \mathbb{U}_{\mathbb{X}_{N-k}}^{k}(x)$. Now, from Assumption 3.6 applied to $V_{N-k}$ we can conclude (again using $V_{N}\left(x^{e}\right)=0$ )

$$
\left|V_{N-k}\left(x_{u_{\varepsilon}}(k, x)\right)\right| \leq \gamma_{V}(\sigma(K)) .
$$

Moreover, Lemma 4.5 and the bound on $\lambda$ yield

$$
\begin{aligned}
V_{\infty}^{u c}(x)+\varepsilon & \geq J_{k}^{u c}\left(x, u_{\varepsilon}\right)+V_{\gamma}\left(x_{u_{\varepsilon}}(k, x)\right) \\
& \geq J_{k}^{u c}\left(x, u_{\varepsilon}\right)-\gamma_{\lambda}(\sigma(K))
\end{aligned}
$$

Together with the dynamic programming principle these inequalities imply

$$
\begin{aligned}
V_{N}(x) & =\inf _{u \in \mathbb{U}_{\mathbb{X}_{N-k}}^{k}(x)}\left\{J_{k}^{u c}(x, u)+V_{N-k}\left(x_{u}(k, x)\right)\right\} \\
& \leq J_{k}^{u c}\left(x, u_{\varepsilon}\right)+V_{N-k}\left(x_{u_{\varepsilon}}(k, x)\right) \\
& \leq V_{\infty}^{u c}(x)+\gamma_{V}(\sigma(K))+\gamma_{\lambda}(\sigma(K))+\varepsilon .
\end{aligned}
$$

Since $\varepsilon>0$ was arbitrary, this proves the assertion for $\delta(N)=\gamma_{V}(\sigma(\lfloor N / 2\rfloor))+\gamma_{\lambda}(\sigma(\lfloor N / 2\rfloor))$.

Since $x^{e}$ is asymptotically stable for the MPC closed loop trajectories, the closed loop solutions particularly converge towards $x^{e}$ as $k \rightarrow \infty$. More precisely, given a time $K$, by Theorem 4.1 the solutions are guaranteed to satisfy $x_{\mu_{N}}(k, x) \in \bar{B}_{\kappa}\left(x^{e}\right)$ for all $k \geq K$ and $\kappa=\beta\left(\left\|x-x^{e}\right\|, K\right)$ for $\beta$ from Theorem 4.1. The time $\operatorname{span}\{0, \ldots, K-1\}$ during which the system is (possibly) outside $\bar{B}_{\kappa}\left(x^{e}\right)$ is called the transient time and the related finite horizon functional $J_{K}^{u c}(x, u)$ is called the transient performance. The next theorem now shows that among all possible trajectories from $x$ to $\bar{B}_{\kappa}\left(x^{e}\right)$, the MPC closed loop has the best transient performance up to error terms vanishing as $K \rightarrow \infty$ and $N \rightarrow \infty$. We remark that unlike the previous theorem here we do not need to assume $\ell\left(x^{e}, u^{e}\right)=0$.
Theorem 5.2: Let Assumptions 3.1, 3.3, 3.4 and 3.6 hold. Then there exist $\delta_{1}, \delta_{2} \in \mathcal{L}$ such that for all all $x \in \mathbb{X}_{N}$ the inequality

$$
J_{K}^{c l}\left(x, \mu_{N}\right) \leq \inf _{u \in \mathbb{U} \bar{K}_{\kappa}\left(x^{e}\right)}(x)=J_{K}^{u c}(x, u)+\delta_{1}(N)+\delta_{2}(K)
$$

holds with $\kappa=\beta\left(\left\|x-x^{e}\right\|, K\right)$ and $\beta$ from Theorem 4.1. Proof: We can without loss of generality assume $\ell\left(x^{e}, u^{e}\right)=$ 0 because the claimed inequality is invariant under adding constants to $\ell$. Moreover, similar to the proof of the previous theorem is is sufficient to prove the inequality for all sufficiently large $K$ and $N$, because by boundedness of all functions involved for small $N$ and $K$ the inequality can always be achieved by choosing $\delta_{1}(N)$ and $\delta_{2}(K)$ sufficiently large. As in the first step of the previous proof we obtain $\left|V_{N}\left(x_{\mu_{N}}(K, x)\right)\right| \leq \gamma_{V}(\sigma(K))$. It is thus sufficient to show the existence of $\delta_{1}, \tilde{\delta}_{2} \in \mathcal{L}$ with

$$
V_{N}(x) \leq \inf _{u \in \mathbb{U}_{\kappa}^{K}(x)} J_{K}^{u c}(x)+\delta_{1}(N)+\tilde{\delta}_{2}(K)
$$

for all $x \in \mathbb{X}_{N}$ because then the assertion follows from (10) with $\delta_{2}=\gamma_{V} \circ \sigma+\tilde{\delta}_{2}$.

To this end, consider $\sigma$ from Lemma 4.6(c), which we apply with $P=\lfloor N / 2\rfloor$ and pick $u_{\varepsilon} \in \mathbb{U}_{\bar{B}_{\kappa}\left(x^{e}\right)}^{K}(x)$ with $J_{K}^{u c}\left(x, u_{\varepsilon}\right) \leq \inf _{u \in \mathbb{U}_{\frac{K}{B_{K}(x e)}}(x)} J_{K}^{u c}(x, u)+\varepsilon$ with an arbitrary but fixed $\varepsilon \in(0,1]$. This yields the existence of $k \in$ $\{0, \ldots,\lfloor N / 2\rfloor\}, k \leq K-1$ with $\left\|x_{u}(k, x)-x^{e}\right\| \leq$ $\sigma(\min \{P, K-1\})$. Since $u_{\varepsilon}$ steers $x$ to $\bar{B}_{\kappa}\left(x^{e}\right)$, the shifted sequence $u_{\varepsilon}(k+\cdot)$ lies in $\mathbb{U}_{\bar{B}_{k}\left(x^{e}\right)}^{K-k}\left(x_{u_{\varepsilon}}(k, x)\right)$, implying that this set is non empty. Hence, we can apply Lemma 4.6(b) in order to conclude $J_{K-k}^{u c}\left(x_{u_{\varepsilon}}(k, x), u_{\varepsilon}(k+\cdot)\right) \geq$ $-\gamma_{\lambda}(\sigma(\min \{N, K-1\}))-\gamma_{\lambda}(\kappa)$. This implies

$$
\begin{aligned}
& \quad \inf _{u \in \mathbb{U}_{\left.\bar{B}_{\kappa(x}{ }^{e}\right)}}(x) \\
& \quad J_{K}^{u c}(x, u)+\varepsilon \geq J_{K}^{u c}\left(x, u_{\varepsilon}\right) \\
& \quad=J_{k}^{u c}\left(x, u_{\varepsilon}\right)+J_{K-k}^{u c}\left(x_{u_{\varepsilon}}(k, x), u_{\varepsilon}(k+\cdot)\right) \\
& \quad \geq J_{k}^{u c}\left(x, u_{\varepsilon}\right)-\gamma_{\lambda}(\sigma(\min \{N, K-1\}))-\gamma_{\lambda}(\kappa)
\end{aligned}
$$

Moreover, by choosing $N$ and $K$ sufficiently large we can ensure $\sigma(\min \{P, K-1\})<\eta$ for $\eta$ from Assumption 3.3(b), implying $u_{\varepsilon} \in \mathbb{U}_{\mathbb{X}_{Q}}^{k}(x)$ for all $Q \geq N_{0}$ and $N_{0}$ from Assumption 3.3(b). Particularly, choosing $N \geq 2 N_{0}$ implies $N-k \geq N_{0}$ and thus $u_{\varepsilon} \in \mathbb{U}_{\mathbb{X}_{N-k}^{k}}^{k}(x)$.

Using this relation, the inequality derived above, the dynamic programming principle and Assumption 3.6 for $V_{N-k}$ we obtain

$$
\begin{aligned}
& \quad V_{N}(x)=\inf _{u \in \mathbb{U}_{\mathbb{X}_{N-k}}^{k}(x)}\left\{J_{k}^{u c}(x, u)+V_{N-k}\left(x_{u}(k, x)\right)\right\} \\
& \leq J_{k}^{u c}\left(x, u_{\varepsilon}\right)+V_{N-k}\left(x_{u_{\varepsilon}}(k, x)\right) \\
& \leq \inf _{u \in \mathbb{U}_{\bar{B}_{\kappa}\left(x^{e}\right)}}(x) \\
& \quad J_{K}^{u c}(x, u)+\gamma_{V}(\sigma(\min \{P, K-1\})) \\
& \quad+\gamma_{V}(\kappa)+\gamma_{\lambda}(\sigma(\min \{P, K-1\}))+\gamma_{\lambda}(\kappa)+\varepsilon .
\end{aligned}
$$

This shows the desired inequality (11) for

$$
\delta_{1}(N)=\gamma_{V}(\sigma(\lfloor N / 2\rfloor))+\gamma_{\lambda}(\sigma(\lfloor N / 2\rfloor))
$$


and, using the choice of $\kappa$,

$$
\begin{aligned}
\tilde{\delta}_{2}(K)= & \gamma_{V}(\sigma(K))+\gamma_{\lambda}(\sigma(K)) \\
& +\gamma_{V}(\beta(M, K))+\gamma_{\lambda}(\beta(M, K))
\end{aligned}
$$

with $M=\sup _{x, y \in \mathbb{X}}\|x-y\|$ and $\beta$ from Theorem 4.1.

Remark 5.3: In the analogous statement for MPC without terminal conditions (Theorem 4.1 in [9]), the respective inequality - translated to the notation used in this paper - reads

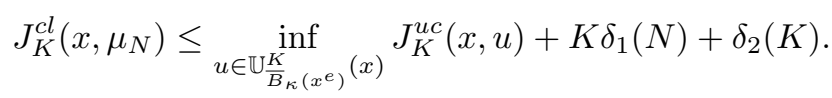

Thus, the benefit of the terminal conditions is to avoid the factor $K$ in front of the error term depending on $N$. Particularly, the terminal conditions ensure that for fixed $N$ the error bound does not degenerate as $K \rightarrow \infty$.

\section{EXAMPLE}

We illustrate our results with a simple 1d example from [7] with dynamics and stage cost

$$
x(k+1)=2 x(k)+u(k), \quad \ell(x, u)=u^{2}
$$

and $\mathbb{Y}=[-2,2] \times[-3,3]$. Hence, the control objective is to keep the system state inside $\mathbb{X}=[-2,2]$ with minimal control effort. One checks that the system is strictly disipative with storage function $\lambda(x)=-x^{2} / 2$ and that $x^{e}=0$ is the (unique) optimal equilibrium with control value $u^{e}=0$. We compare the values $J_{K}^{c l}\left(x, \mu_{N}\right)$ for initial condition $x=2$ for the MPC scheme with terminal constraint set $\mathbb{X}_{0}=\{0\}$ and terminal cost $V_{f}\left(x^{e}\right)=0$ with the scheme without any terminal constraints and costs as considered in [7]. Figure 1 shows the respective values $J_{K}^{c l}\left(x, \mu_{N}\right)$ for fixed $N=5$ and $K=1, \ldots, 25$. One sees that for small $K$ the controller obtained without terminal conditions has advantages, but since one of the error terms without terminal constraints grows linearly in $K$, cf. Remark 5.3, for growing $K$ the controller computed with terminal constraints performs better and, in fact, converges to $J_{\infty}^{c l}\left(x, \mu_{N}\right)$.

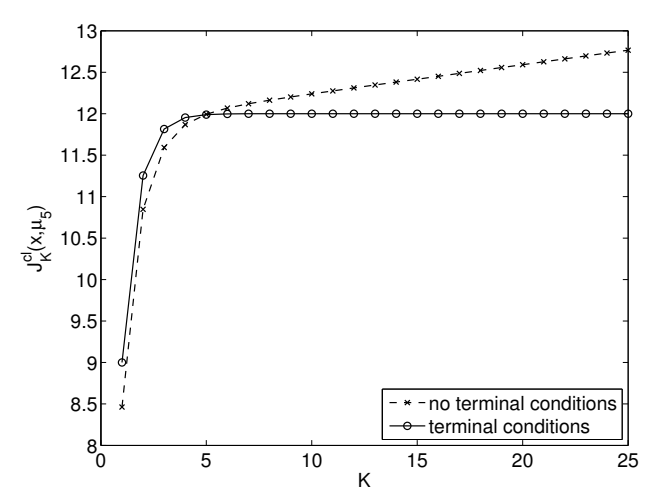

Fig. 1. Performance of MPC controllers computed with and without terminal constraints for fixed $N=5$ and varying $K=1, \ldots, 25$

Figure 2 shows the respective values $J_{K}^{c l}\left(x, \mu_{N}\right)$ for fixed $K=20$ and $N=1, \ldots, 10$. Here one sees that in this examples the terminal constraints yield significant improvement for small $N$, while for larger $N$ the difference in performance is almost negligible.

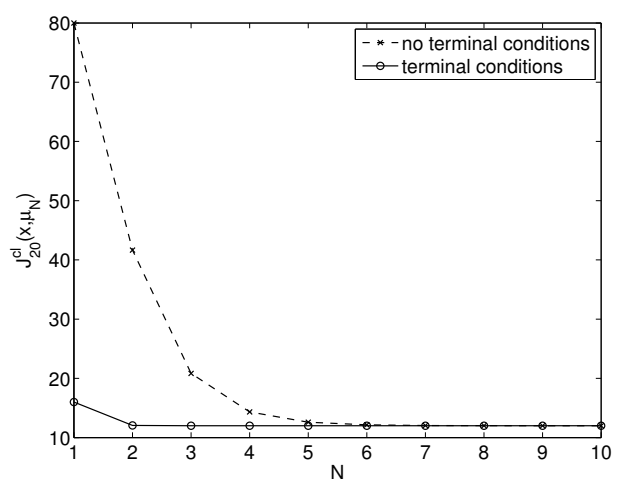

Fig. 2. Performance of MPC controllers computed with and without terminal constraints for fixed $K=20$ and varying $N=1, \ldots, 10$

\section{CONCLUSION}

We have considered economic MPC schemes under the usual assumptions ensuring existence and asymptotic stability of an optimal steady state. For these schemes we have shown that beyond the previously established averaged optimality, the MPC closed loop trajectories also exhibit approximately optimal non-averaged infinite horizon and transient performance.

\section{REFERENCES}

[1] R. Amrit, J. B. Rawlings, and D. Angeli, "Economic optimization using model predictive control with a terminal cost," Annual Rev. Control, vol. 35, pp. 178-186, 2011.

[2] D. Angeli, R. Amrit, and J. B. Rawlings, "Receding horizon cost optimization for overly constrained nonlinear plants," in Proceedings of the 48th IEEE Conference on Decision and Control - CDC 2009, Shanghai, China, 2009, pp. 7972-7977.

[3] — , "On average performance and stability of economic model predictive control," IEEE Trans. Autom. Control, vol. 57, no. 7, pp. 1615-1626, 2012.

[4] D. A. Carlson, A. B. Haurie, and A. Leizarowitz, Infinite horizon optimal control - Deterministic and Stochastic Systems, 2nd ed. Berlin: Springer-Verlag, 1991.

[5] T. Damm, L. Grüne, M. Stieler, and K. Worthmann, "An exponential turnpike theorem for dissipative discrete time optimal control problems," SIAM J. Control Optim., vol. 52, pp. 1935-1957, 2014.

[6] M. Diehl, R. Amrit, and J. B. Rawlings, "A Lyapunov function for economic optimizing model predictive control," IEEE Trans. Autom. Control, vol. 56, pp. 703-707, 2011.

[7] L. Grüne, "Economic receding horizon control without terminal constraints," Automatica, vol. 49, pp. 725-734, 2013.

[8] L. Grüne and J. Pannek, Nonlinear Model Predictive Control. Theory and Algorithms. London: Springer-Verlag, 2011.

[9] L. Grüne and M. Stieler, "Asymptotic stability and transient optimality of economic MPC without terminal conditions," J. Proc. Control, vol. 24, no. 8, pp. 1187-1196, 2014.

[10] M. A. Müller, D. Angeli, and F. Allgöwer, "On necessity and robustness of dissipativity in economic model predictive control," IEEE Trans. Autom. Control, 2015, to appear, DOI 10.1109/TAC.2014.2361193 